\title{
Continuum Computational Fluid Dynamics model of heat transfer in monolith converter
}

\author{
This article was published in the following Dove Press journal: \\ Energy and Emission Control Technologies \\ 10 June 2013 \\ Number of times this article has been viewed
}

\begin{abstract}
Atul Pant ${ }^{1}$
Thiyagarajan

Paramadayalan ${ }^{2}$

'Global General Motors R\&D, India Science Lab, GM Tech Center (India), Bangalore, India; ${ }^{2} \mathrm{GM}$ PowertrainIndia, Engine CAE, GM Tech Center (India), Bangalore, India
\end{abstract}

\begin{abstract}
A continuum model representation of transient heat transfer in monoliths with square channels requires solving separate energy balance for gas and solid domains. Comparison of continuum model predictions with results of a full three-dimensional model of distributed square channels shows that besides adequate estimation of effective radial thermal conductivity, heat flux at boundary also needs to be properly assigned. It is shown that solving separate energy balances for solid and fluid domains requires splitting the total boundary heat flux between the two domains. The heat flux can be split based on thermal resistance of the fluid and solid domain in the continuum model. Heat transfer in a monolith with 1024 square channels is simulated using the proposed continuum approach and the direct approach considering each individual channel. The continuum model predicts the radial variation in temperature to within $6^{\circ} \mathrm{C}$ of that obtained in the actual channel model. Using the model to simulate the heating up of the monolith with hot inlet gas and heat loss at the boundary shows that the heat loss at the monolith boundary does not penetrate to more than three channels near the wall.
\end{abstract}

Keywords: monolith reactors, heat conduction, heat transfer, mathematical modeling, numerical analysis, porous media

\section{Introduction}

Catalytic converters are widely used for reducing tail pipe emissions from a car. A typical converter consists of a monolith with multiple square channels with catalyst on the channel walls. The typical size of a channel is of the order of $1 \mathrm{~mm}$ with wall thickness of $\sim 0.1 \mathrm{~mm} .{ }^{1}$ Modeling radial and axial temperature distribution in the monolith as a result of heating from exhaust gas and exothermic reactions is important for predicting cold start emissions. Kolaczkowski et $\mathrm{al}^{2}$ made the first attempt to model steady-state radial heat transfer in monolithic converters by considering channels with finite wall thickness. More recently, Kumar and Mazumdar ${ }^{3}$ simulated heat transfer with flow and reaction in a full three-dimensional (3D) model of channels. The authors found that computational time increased significantly with the number of channels considered in the computation domain. Traditionally, to reduce the computational time, most 3D models approximate the monolith as a continuum and model it as a porous medium. ${ }^{4,5}$ The continuum model is an effective way to solve mathematical representations of an actual system with less computational time.

Most Computational Fluid Dynamics (CFD) solvers assume a thermal equilibrium between fluid and solid in the porous medium. ${ }^{6}$ Equality of solid and gas temperature is an acceptable assumption under steady-state, nonreacting conditions. However, to accurately predict the rise in converter temperature during transient and exothermic submit your manuscript | www.dovepress.com

Dovepress

http://dx.doi.org./10.2147/EECT.S44770
Energy and Emission Control Technologies 2013:I I-13

(C) 2013 Pant and Paramadayalan, publisher and licensee Dove Medical Press Ltd. This is an Open Access article which permits unrestricted noncommercial use, provided the original work is properly cited. 
reactions conditions, the solid and gas phase temperatures have to be solved separately. ${ }^{7-9}$ Solving separate energy equations for gas and solids requires the correct specification of thermal conductivity and wall boundary conditions. Several formulations have been proposed in the past for calculating the effective thermal conductivity of monoliths. Liu ${ }^{10}$ and Zygourakis ${ }^{9}$ derived an effective conductivity based on the equivalent continuum approach, which is widely used by CFD models. ${ }^{4,8,11}$ However, the equivalent continuum approach breaks down for the limiting case when the total solid fraction in the porous medium tends to zero or one. Alternative formulations based on a thermal resistance circuit have been proposed by Groppi and Tronconi, ${ }^{12}$ as well as by Hayes et al. ${ }^{13}$ Both of these treatments provide a single expression for the effective thermal conductivity of the porous medium representing the monolith, but they do not have separate expressions for the solid and fluid phases. Hayes et $\mathrm{al}^{13}$ assumed that the effective gas phase conductivity would be zero and assigned the total effective conductivity to the solid. The effect of the solid network structure on the effective conductivity of the individual domain is not considered. On the other hand, Sahraoui and Kaviany, ${ }^{14}$ in their work on distributed solids in a fluid medium, formulated separate expressions for the effective conductivity of both solids and gas using an equivalent thermal circuit approach.

While solving separate energy equations for the distributed and continuous phases of a networked structure like a monolith, the total heat flux at the external boundary should be adequately proportioned between the two new boundaries for each of the phases in the continuum model. Initial multidimensional mathematical models applied the total heat loss boundary condition on the solid phase. ${ }^{7,9,15}$ In these cases, the thermal conductivity of the gas medium was neglected, and effective radial conductivity was considered for the solid medium. A similar approach was followed by recent CFD models. ${ }^{1,16}$ In all of these cases, the solid phase, which has the higher thermal conductivity, was the continuous medium. Therefore, it physically makes sense to apply the heat flux boundary condition on the solid phase energy balance since the solid is in contact with the external boundary. However, it is not very clear if similar boundary condition specifications will be valid for instances when the thermal conductivity of the continuous medium is lower than that of the distributed medium. To our knowledge, no validated treatment for the heat flux condition at the external boundary of the distributed and continuous medium is available.

In this paper, we outline the development and validation of the $3 \mathrm{D}$ continuum model for heat transfer in a monolith with square channels. We have established guidelines for choosing physically consistent properties and boundary conditions when using a continuum model with two separate domains. The effective thermal conductivity of both the gas and solid phases is determined based on the thermal network. A self-consistent approach is developed to derive the appropriate heat flux boundary condition on the external surface of both the fluid and solid domains. This continuum approach is referred to as the "porous model" in the text. The porous model is validated by comparing it with the simulation results of a complete, 3D model of a monolith with 1024 square channels. The complete $3 \mathrm{D}$ model of these channels is referred to as the "channel model" in the text.

\section{Model development Channel model}

A $75 \mathrm{~mm}$ long section of a monolith with 1024 square channels and a $0.14 \mathrm{~mm}$ thick wall is modeled in Star-CD, version 3.26 (Computational Dynamics Ltd, NY,USA). ${ }^{6}$ As shown in Figure 1, besides the inlet and outlet, the geometry is bounded by walls on two sides and assumes symmetry on the other two sides. The symmetry condition enables simulation of the 1024 channels using only 256 channels in the CFD model. The model geometry also includes $75 \mathrm{~mm}$ long, fluid only zones at the monolith inlet and outlet. This is used to model the flow of gas in and out of the monolith. Typically, the catalyst is coated on the walls of the catalytic monolith in a layer referred to as the washcoat. In this model, the washcoat and channel wall are combined and modeled as a single solid domain. Approximately $21 \%$ of the solid wall is assumed to be washcoat for the purpose of calculating the wall's thermal properties. The momentum and energy equation for fluid are

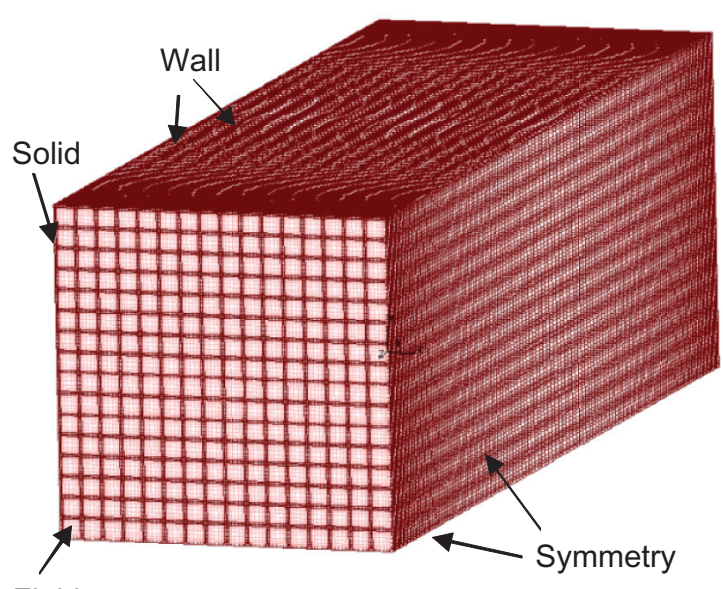

Fluid

Figure I Representation of the I/4 geometry with 256 channels.

Note: The extended fluid domain for the inlet and outlet are not shown for clarity. 
solved in the fluid region. The energy equation is solved in the solid domain with conjugate heat transfer between the solid and fluid domains.

\section{Porous model}

In the continuum approach, the monolith section corresponding to 1024 channels is modeled as porous medium in Star$\mathrm{CD}$, with the outer dimensions being similar to the channel model described in the channel model. Star-CD implementation assumes a thermal equilibrium between the gas and solid temperature in a porous medium; therefore, a separate, parallel, solid domain is considered to solve the solid temperature, as shown in Figure 2. The gas phase temperature and the pressure drop are solved in the porous medium. The solid domain size and grid is the same as the porous domain, with one-to-one correspondence between the grid cells. The heat transfer between fluid and solid regions is modeled by using explicitly coupled source terms in the energy equations of the two separate domains. The governing equations in the porous model are described below.

Star-CD solves the pressure drop in the porous domain as an additional source term in the momentum equation as follows,

$$
K_{i} v_{i}=\frac{\partial p}{\partial \xi_{i}}
$$

where $\xi_{i}(I=1,2,3)$ represents the (mutually orthogonal) orthotropic directions, $K_{i}$ is the porous resistance and $v_{i}$ is the superficial velocity in direction $\xi_{i}$. The resistance $K_{i}$ is assumed to be a function of the superficial velocity magnitude $|v|$ of the form

$$
K_{i}=\alpha_{i}|v|+\beta_{i}
$$

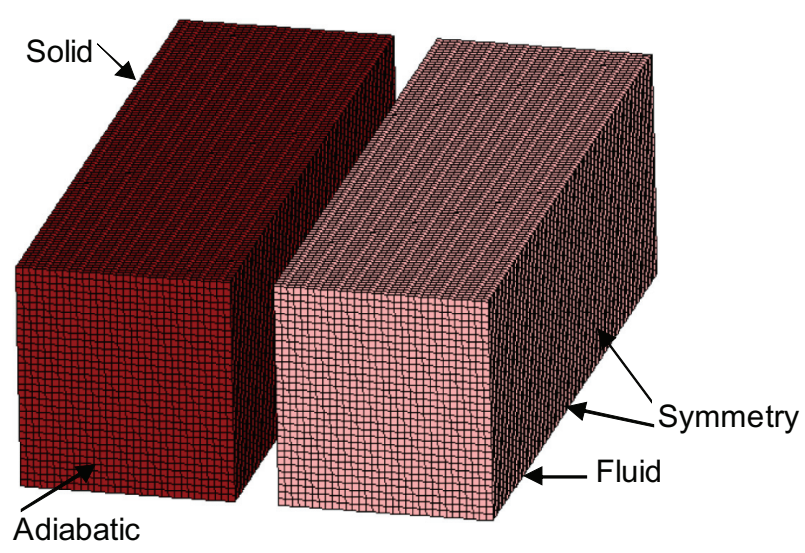

Figure 2 Porous model representation of the I/4 geometry shown in Figure I. where $\alpha_{i}$ and $\beta_{i}$ are resistance coefficients calculated from monolith characteristics following the pressure drop expression given by Kays and London, ${ }^{17}$

$$
\alpha_{i}=\frac{\rho}{2 L \varepsilon^{2}}\left(K_{c}+K_{e}\right) \quad \beta_{i}=\frac{2 f_{\text {shape }} \mu}{\varepsilon D_{h}^{2}}
$$

where, $\varepsilon$ is porosity, $\rho$ is fluid density, $D_{h}$ is hydraulic diameter, $L$ is the length, $\mu$ is viscosity, $f_{\text {shape }}$ is friction shape factor, $K_{c}$ is the entrance pressure loss coefficient, and $K_{e}$ is the exit pressure loss coefficient. The calculated $\alpha_{i}$ and $\beta_{i}$ are specified only in the axial direction because the flow in the monolith channels is unidirectional. The transverse coefficients are assigned large values $\left(10^{8}\right)$ to suppress the flow in radial directions. ${ }^{18}$ Star-CD solves for a single temperature in the porous medium. This equation has been modified, as shown below, to represent the fluid phase energy balance in the channels. A source term is added to account for the heat transfer between the gas and solid walls in the converter.

$$
\begin{aligned}
& \varepsilon \rho_{g} C p_{g} \frac{\partial T_{g}}{\partial t}+\frac{w}{A} C p_{g}\left(\frac{\partial T_{g}}{\partial x}+\frac{\partial T_{g}}{\partial y}+\frac{\partial T_{g}}{\partial z}\right) \\
& =k_{e f f}\left(\left(\frac{\partial^{2} T_{g}}{\partial x^{2}}\right)+\left(\frac{\partial^{2} T_{g}}{\partial y^{2}}\right)+\left(\frac{\partial^{2} T_{g}}{\partial z^{2}}\right)\right)-h S\left(T_{g}-T_{s}\right)
\end{aligned}
$$

Here, $T_{s}$ is the solid phase temperature and $T_{g}$ is the fluid temperature. Gas density $\rho_{g}$ is a function of temperature based on the ideal gas law. Moreover, $\varepsilon$ is porosity and $k_{\text {eff }}$ is the effective gas phase conductivity. Current Star-CD implementation does not allow anisotropic conductivity specification for porous medium. Therefore, equal conductivities are specified in all directions. This is not a severe assumption because contribution from gas phase conduction to overall heat transfer is usually small. The effective conductivity, $k_{\text {eff }}$, depends on the geometry and is discussed in detail in the following section.

The solid phase energy equation, as shown below, is solved separately in the solid domain.

$$
\begin{aligned}
(1-\mathcal{E}) \rho_{s} C p_{s} \frac{\partial T_{s}}{\partial t}= & k_{r s, e f f}\left(\frac{\partial^{2} T_{s}}{\partial x^{2}}\right)+k_{r s, e f f}\left(\frac{\partial^{2} T_{s}}{\partial y^{2}}\right) \\
& +k_{a s, e f f}\left(\frac{\partial^{2} T_{s}}{\partial z^{2}}\right)+h S\left(T_{g}-T_{s}\right)
\end{aligned}
$$

Volume averaged properties (Equations 6 to 8) based on washcoat, $f_{w c}$, and substrate, $f_{s b}$ volume fractions are used for the solid domain ${ }^{19}$ 


$$
\begin{gathered}
\rho_{s} C p_{s}=f_{s b} \rho_{s b} C p_{s b}+f_{w c} \rho_{w c} C p_{w c} \\
k_{s}=f_{s b} k_{s b}+f_{w c} k_{w c} \\
C p_{s b}=1071+0.156 T_{s}-\frac{3.435 \times 10^{7}}{T_{s}^{2}} \text { and } C p_{w c}=1000
\end{gathered}
$$

The axial conductivity, $k_{a s, e f f}$, in Equation 5 is given by $k_{a s, e f f}=(1-\varepsilon) k_{s}$, and the effective radial conductivity, $k_{r s, e f f}$, is calculated based on the geometry as discussed in the following sections. The term, $h S\left(T_{g}-T_{s}\right)$, accounts for the heat transfer between fluid and solid. The heat transfer coefficient is determined by the following equation:

$$
h=\frac{N u}{D_{h}} k_{g}
$$

where $N u$ is the Nusselt number, assumed to be 3 for fully developed flow, and $D_{h}$ is the hydraulic diameter of the channel. The thermal conductivity of the gas, $k_{g}$, is given by Equation $10^{20}$

$$
k_{g}\left(T_{g}\right)=2.66 \times 10^{-4} T_{g}^{0.805}
$$

\section{Effective transverse thermal conductivity of square channels}

Researchers using the continuum approach for modeling networked structures have used effective transverse thermal conductivity calculated based on the thermal resistance network. ${ }^{12,13}$ The thermal resistance circuit for a unit cell of a monolith consisting of square channels can be constructed in two methods. Hayes et $\mathrm{al}^{13}$ calculated the effective resistance considering that all forms of resistance are in parallel and compared it with the series and parallel resistances approach formulated by Groppi and Tronconi. ${ }^{12}$ Equation 11 shows the effective thermal conductivity derived using the Hayes et al: ${ }^{13}$ whereas equation 12 is the effective conductivity expression obtained using Groppi and Tronconi. ${ }^{12}$

$$
k_{r, e f f}=R_{e q}^{-1}=k_{s}\left[1-\sqrt{\varepsilon}+\frac{\sqrt{\varepsilon}}{1-\sqrt{\varepsilon}+\left(k_{s} / k_{f}\right) \sqrt{\varepsilon}}\right]
$$

$$
k_{r, e f f}=\frac{1}{R_{e q}}=k_{s}\left[1-\sqrt{\varepsilon}+\frac{\sqrt{\varepsilon}}{1-\sqrt{\varepsilon}+\left(k_{f} / k_{s}\right) \sqrt{\varepsilon}}\right]^{-1}
$$

A steady-state heat transfer problem in actual square channels is solved in Star-CD. The predicted temperature profile is used to estimate the effective thermal conductivity, which is compared with the theoretical predictions given by Equations 11 and 12. A small $5 \mathrm{~mm}$ section of the 256 square channel model (described earlier) was used for this simulation. Models with cell densities ranging from 300 to $800 \mathrm{cpsi}$ and with a solid thermal conductivity of $0.1,1$, and $10 \mathrm{~W} / \mathrm{mK}$ were simulated using the geometry shown in Figure 3.

A constant heat flux of $300 \mathrm{~W} / \mathrm{m}^{2}$ was applied on the top face, and the bottom face was maintained at a constant temperature of $450 \mathrm{~K}$. All other faces were adiabatic. The model was simulated with different cell densities and solid thermal conductivities to obtain steady-state temperature profiles, as shown in Figure 4. The gradient in steady-state temperature and heat flux $(q)$ at the top face was used to calculate the effective radial thermal conductivity using Equation 13

$$
q=k_{r, e f f} \frac{\Delta T}{L}
$$

where $L$ is the length of the section. The effective conductivity calculated from numerical simulations is compared against the effective conductivity obtained from Equations 11 and

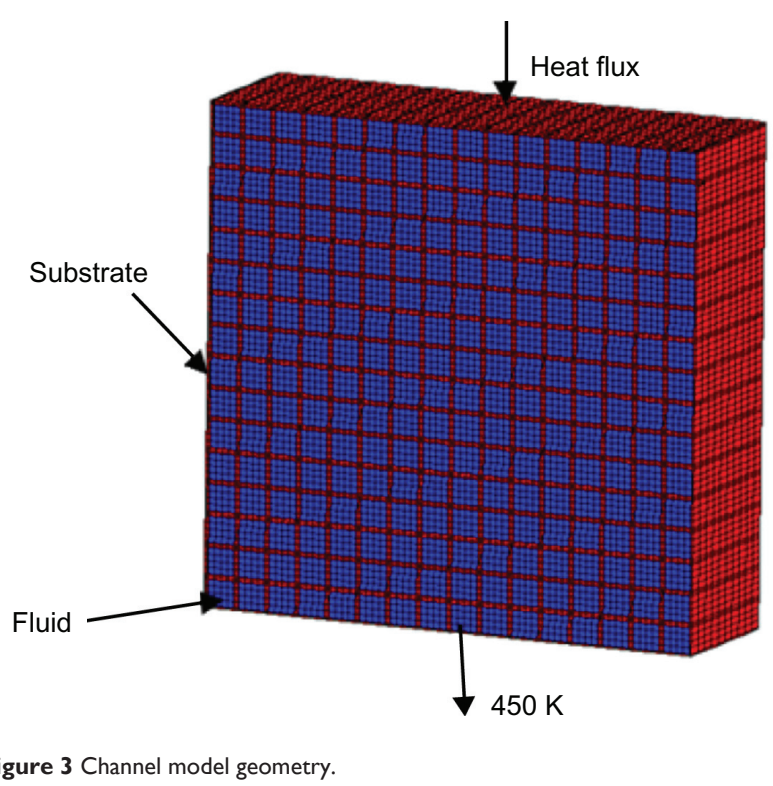




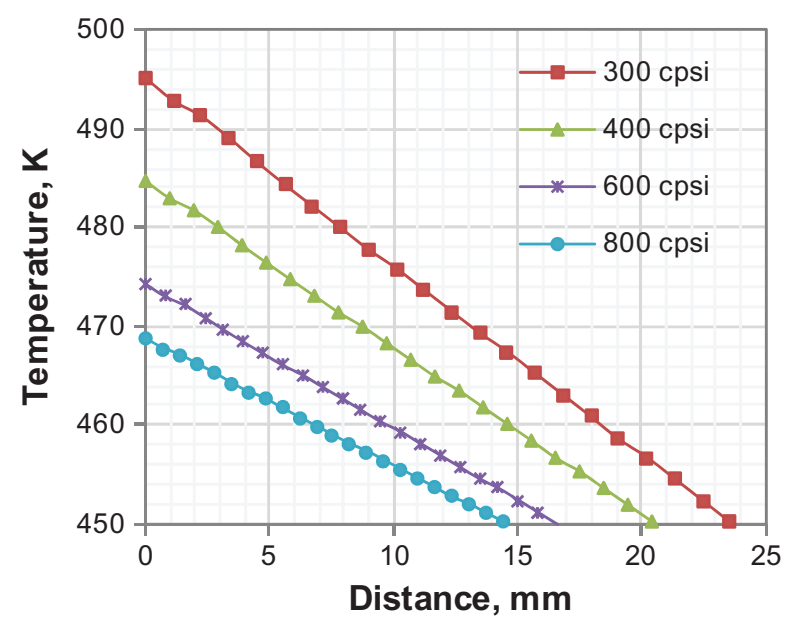

Figure 4 Steady-state temperature profiles. Note: Across channels, solid conductivity of I $\mathrm{Wm}^{-1} \mathrm{~K}^{-1}$.

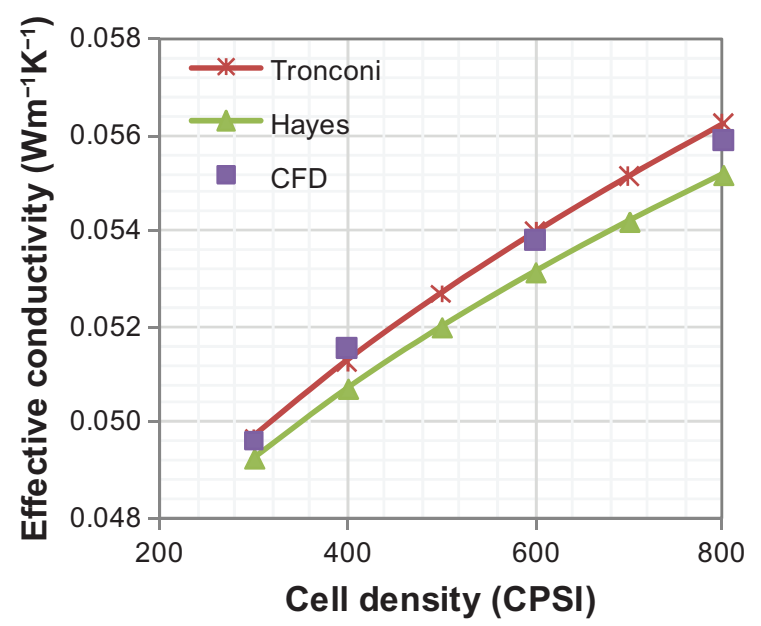

Figure 5 Effective thermal conductivity.

Note: For solid conductivity of $0.1 \mathrm{Wm}^{-1} \mathrm{~K}^{-1}$.

Abbreviation: CFD, computational fluid dynamics.

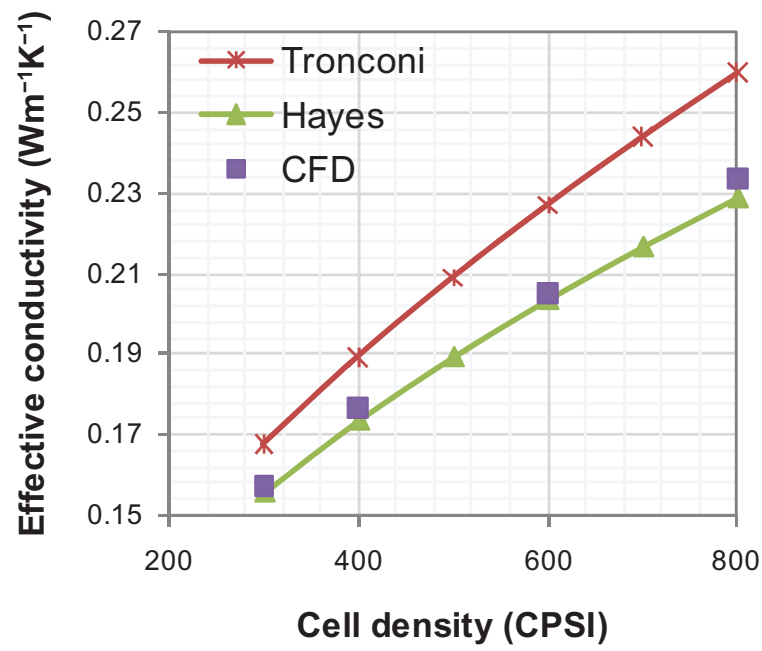

Figure 6 Effective thermal conductivity.

Note: For solid conductivity of $\mathrm{I} \mathrm{Wm}^{-1} \mathrm{~K}^{-1}$.

Abbreviation: CFD, computational fluid dynamics.

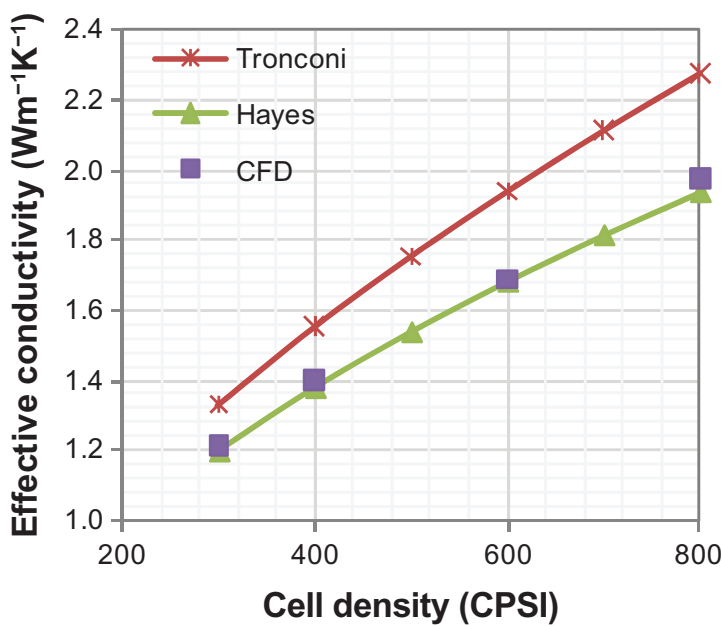

Figure 7 Effective thermal conductivity.

Note: For solid conductivity of $10 \mathrm{Wm}^{-1} \mathrm{~K}^{-1}$.

Abbreviation: CFD, computational fluid dynamics.

12 in Figures 5 through 7. As reported by Hayes et al, ${ }^{13}$ our results also showed that the effective thermal conductivity calculated using the parallel approach is closer to the values estimated by simulation, except at low solid thermal conductivity. At low solid conductivity, the series parallel approach matches the data better; however, the parallel approach also predicts the thermal conductivity within $1.2 \%$. Based on this analysis, the parallel resistance arrangement is chosen to be representative of the solid network in square channels and is used in our analysis to calculate the effective radial thermal conductivity.

\section{Effective thermal conductivity of solid and fluid domain in a porous model}

The effective radial thermal conductivity from the above analysis can be directly used in cases where the catalytic converter is modeled as a single porous domain; however, this is not possible when the solid and gas energy balance are solved separately. Previous studies have assumed that the radial thermal conductivity for solids is equal to the effective thermal conductivity in the radial direction, which has neglected the gas phase radial thermal conductivity. ${ }^{12,13}$ In contrast, Sahraoui and Kaviany ${ }^{14}$ proposed that when solving the solid and gas phase energy balance separately (using a continuum model approach), both phases should have effective radial thermal conductivity. The total effective thermal conductivity is the sum of the effective thermal conductivity of the individual phases as given below:

$$
k_{r, e f f}=k_{s, e f f}+k_{g, e f f} .
$$




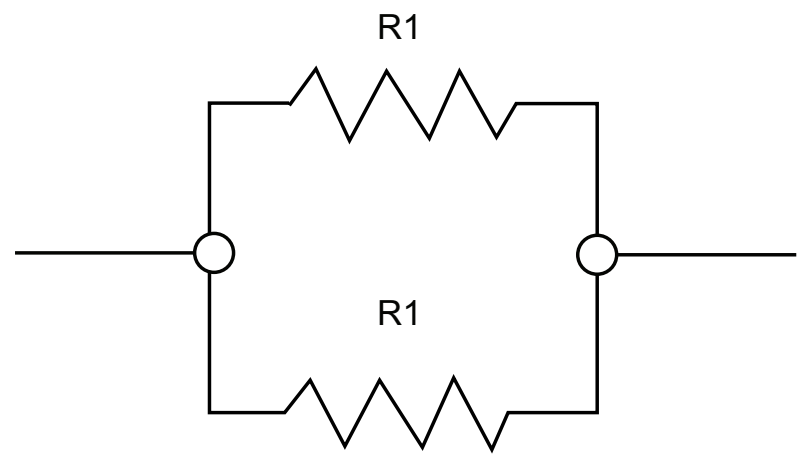

Figure 8 Equivalent circuit for the solid network only.

We have chosen to use an analysis similar to that of Sahraoui and Kaviany. ${ }^{14}$ The effective solid conductivity, $k_{s, e f f}$, is assumed to be equal to the thermal conductivity obtained from a resistance network analysis, assuming that no gas phase is present. In the absence of gas phase, the thermal resistance circuit of a unit cell takes the form shown in Figure 8 , where $\mathrm{R} 1$ is the thermal resistance of the solid.

The equivalent resistance of the circuit is

$$
R_{e q}^{-1}=2 R_{1}^{-1}
$$

therefore, the effective conductivity for the solid-only network is given by Equation 16. This is equivalent to putting $K_{f}=0$ in Equation 11.

$$
k_{s, e f f}=k_{s}(1-\sqrt{\varepsilon})
$$

The corresponding effective thermal conductivity for the gas phase from Equations 14 and 16 is

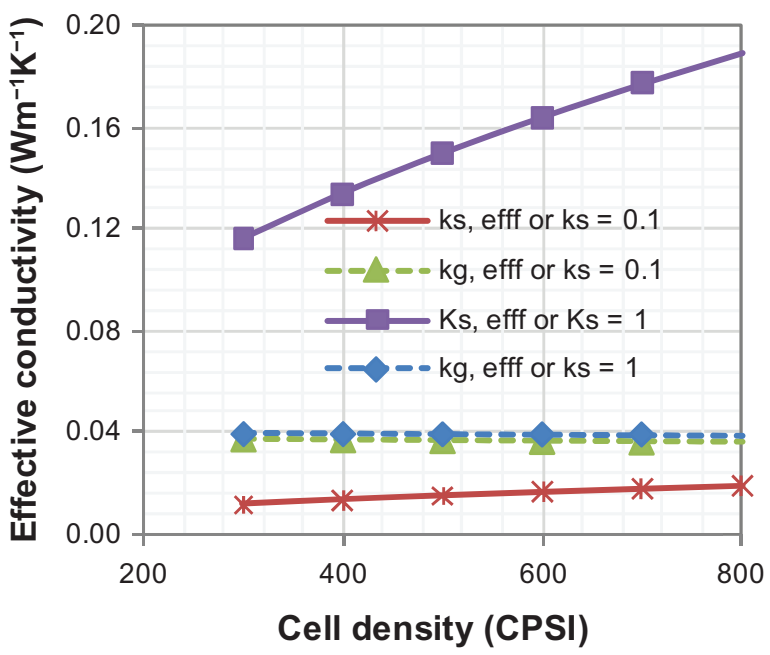

Figure 9 Effective thermal conductivity of gas and solid phases versus cell density.

$$
k_{g, \text { eff }}=k_{s}\left[\frac{\sqrt{\varepsilon}}{1-\sqrt{\varepsilon}+\left(k_{s} / k_{f}\right) \sqrt{\varepsilon}}\right] \text {. }
$$

Figure 9 show the effective radial conductivity of the solid and gas domain calculated using Equations 16 and 17, respectively. These calculations use constant gas conductivity, $k_{g}$, of $0.039 \mathrm{~W} / \mathrm{mK}$. Neglecting the effective radial gas conductivity at high solid conductivity is an acceptable assumption because most of the heat will transfer through the solid domain due to higher thermal conductivity. However, at lower solid conductivity, the effective conductivities of the solid and gas domain are comparable and the assumption of neglecting gas phase radial conductivity should be verified.

\section{Flux boundary condition of solid and fluid domains in the porous model}

To establish the heat flux boundary condition specification at the external surface of the two separate domains in the porous model, the monolith section with 256 channels shown in Figure 3 is modeled using the continuum approach. The geometrical representation is shown in Figure 10. The steadystate heat transfer problem solved in the channel model in section 2.3 is implemented in the continuum model. The gas phase energy balance is solved in the porous domain using radial thermal conductivity obtained from Equation 17. The solid phase energy balance is solved in the coupled parallel solid domain using the radial conductivity obtained from Equation 16.

In the channel model, a constant heat flux $\left(300 \mathrm{~W} / \mathrm{m}^{2}\right)$ is applied on the top face and a constant temperature of $450 \mathrm{~K}$ is applied on the bottom face. In the porous model, this heat flux has to be adequately distributed between the solid and porous domain boundaries because the energy balance is solved separately in the two domains. A methodology to split the heat flux boundary condition using the resistance analogy

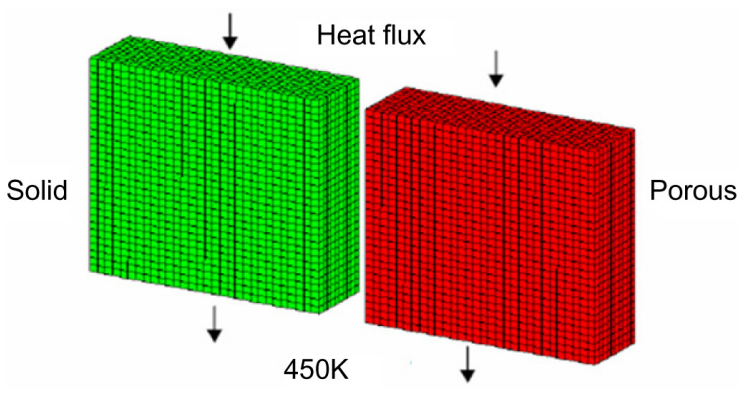

Figure 10 Continuum representation of the channel geometry of Figure 3. 


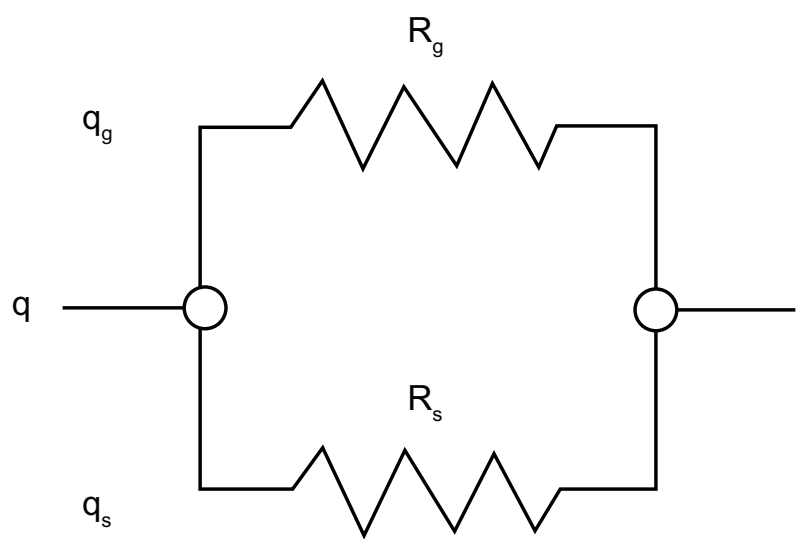

Figure II Equivalent resistance circuit for heat flow from the boundary in the porous model.

is proposed. The equivalent resistance circuit for heat flow is represented as shown in Figure 11.

The resistance for heat flow in the gas and solid phases are given by:

$$
R_{s}=\frac{\Delta l}{k_{s, e f f}}, \quad R_{g}=\frac{\Delta l}{k_{g, e f f}}
$$

where $\Delta l$ is the length of the domain. The corresponding heat fluxes through each domain are given by:

$$
q_{g}=\frac{\Delta T}{R_{g}}, \quad q_{s}=\frac{\Delta T}{R_{s}} .
$$

Using Equations 14, 18, and 19, and knowing that $q=q_{s}+q_{g}$, we can show that

$$
\begin{aligned}
& q_{s}=\frac{k_{s, e f f}}{k_{r, e f f}} q \\
& q_{g}=q-q_{s} .
\end{aligned}
$$

In the continuum model, the heat flux and heat transfer coefficients are divided between the two domains according to Equations 20 and 21, and the steady-state heat transfer is simulated. The temperature profile along the vertical edge of the solid domain is compared with the corresponding channel model profile for different cell densities and solid conductivities in Figures 12 to 14. The steady-state temperature profile of the porous model is within $0.5 \%$ of the one obtained from the channel model simulations.

\section{Impact of the fluid-solid heat transfer coefficient}

The effect of uncertainty in estimating the heat transfer coefficient used in energy Equations 4 and 5 is also investigated.

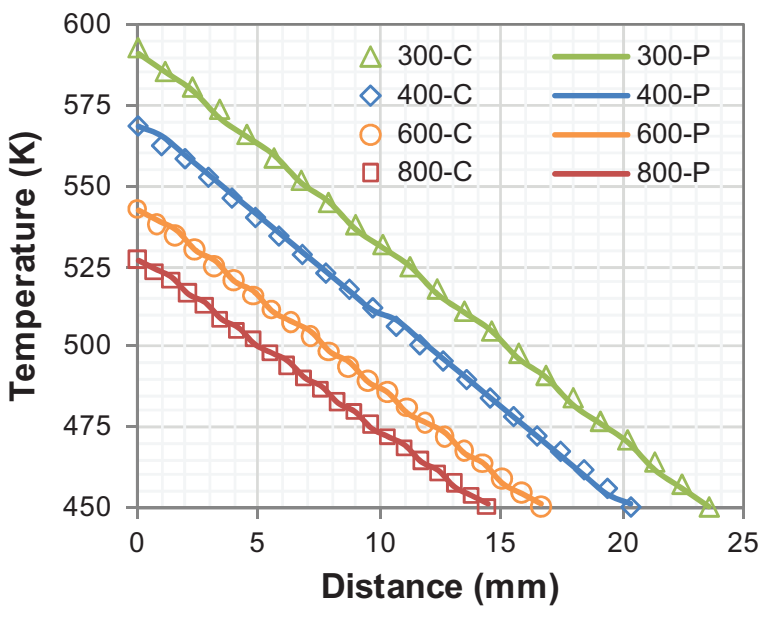

Figure 12 Temperature profile $\left(k_{\mathrm{s}}=0.1 \mathrm{~W} / \mathrm{mK}\right)$.

Note: The numbers in the legend represent cell density in cpsi. Abbreviations: $\mathrm{C}$, channel model; $\mathrm{P}$, porous model.

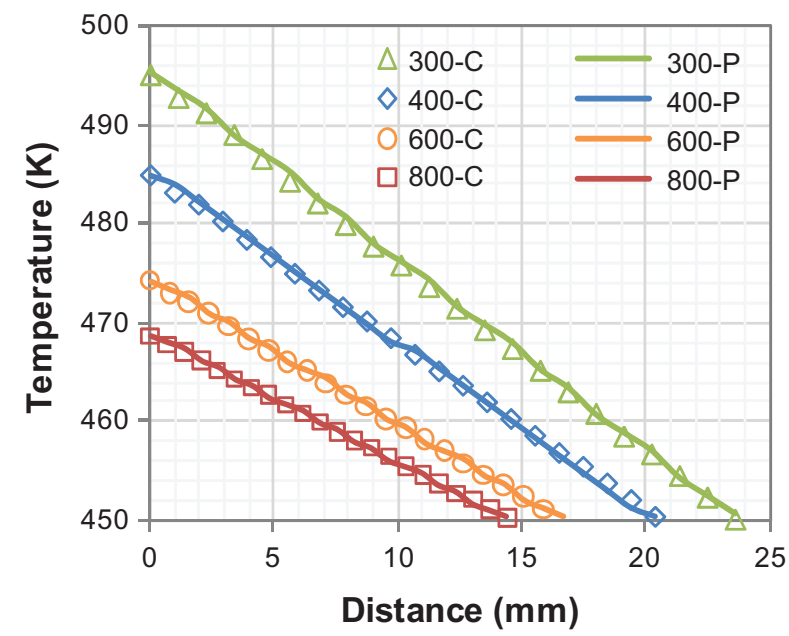

Figure 13 Temperature profile $\left(k_{\mathrm{s}}=1 \mathrm{~W} / \mathrm{mK}\right)$.

Note: The numbers in the legend represent cell density in cpsi. Abbreviations: $\mathrm{C}$, channel model; $\mathrm{P}$, porous model.

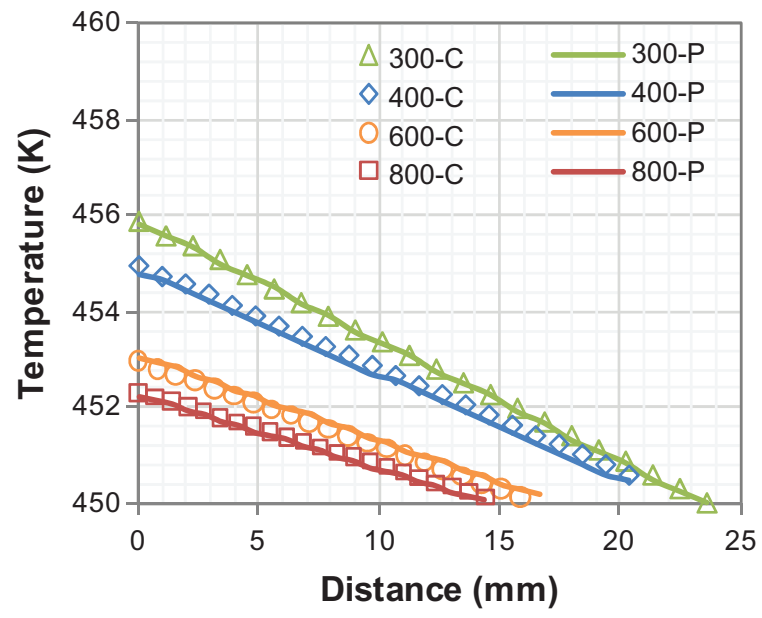

Figure 14 Temperature profile $\left(k_{\mathrm{s}}=10 \mathrm{~W} / \mathrm{mK}\right)$.

Note: The numbers in the legend represent cell density in cpsi.

Abbreviations: $\mathrm{C}$, channel model; $\mathrm{P}$, porous model. 
The simulation results shown in Figures 12 to 14 were repeated for the heat transfer coefficients ranging from $1 \mathrm{~W} / \mathrm{m}^{2} \mathrm{~K}$ (natural convection) to $50 \mathrm{~W} / \mathrm{m}^{2} \mathrm{~K}$ (forced convection). It was observed that when the boundary heat flux is split between the two energy equations based on Equations 20 and 21 , the gas and solid temperature predictions were the same as those predicted by the channel model, irrespective of the heat transfer coefficient values. However, when the boundary heat flux was not proportioned properly between the two phases, the prediction accuracy depended on the heat transfer coefficient value. To illustrate this point the porous model was simulated with modified conditions. In this simulation, the total heat flux at the boundary was only considered in the energy equation for the solid domain. The fluid phase energy equation was solved using adiabatic boundary condition. In addition, the thermal conductivity of the solid domain was considered to be equal to the effective thermal conductivity. A nominal gas thermal conductivity, $\mathrm{k}_{\mathrm{g}}=0.039 \mathrm{~W} / \mathrm{mK}$, was used. It was found that for heat transfer coefficients of $1 \mathrm{~W} / \mathrm{m}^{2} \mathrm{~K}$, the steady-state gas and solid temperatures were not equal, as seen from Figures 15 and 16. The predicted steady-state gas and solid temperatures were equal only at higher heat transfer coefficient values (above $\sim 15 \mathrm{~W} / \mathrm{m}^{2} \mathrm{~K}$ ). From channel model simulations, we have seen that at steady state, gas and solid temperatures are equal for the heat flux problem solved earlier. This dependence of the porous model results on heat transfer coefficients with arbitrary splitting of heat flux at boundaries is more pronounced at lower solid conductivity values. At higher solid conductivity $(10 \mathrm{~W} / \mathrm{mK})$, applying all the heat flux to the solid domain does not alter the results since the amount of heat flux to be attributed to the fluid phase (based on Equation 21) is low (3\% of total). A similar dependence on the heat transfer coefficient is

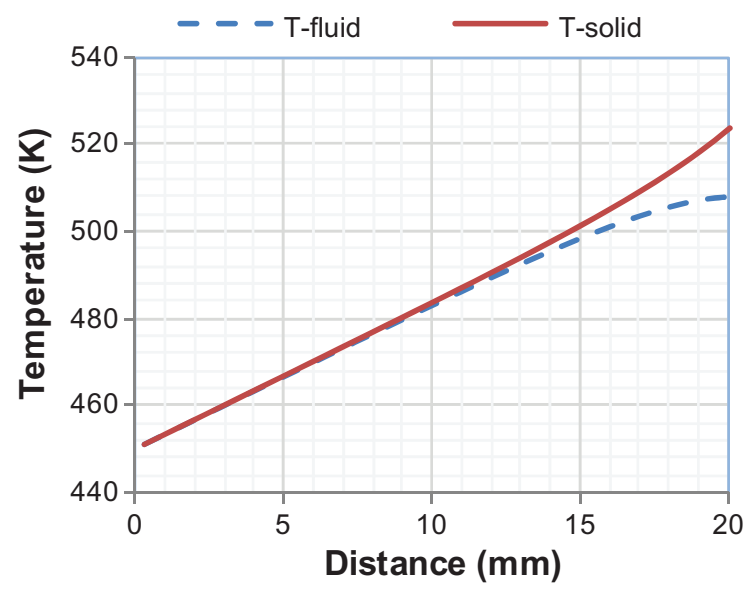

Figure 15 Fluid and solid T profile for $k_{s}=0.1 \mathrm{~W} / \mathrm{mK}$ with $\mathrm{h}=1 \mathrm{~W} / \mathrm{m}^{2} \mathrm{~K}$. Abbreviations: T, temperature; h, heat transfer coefficient.

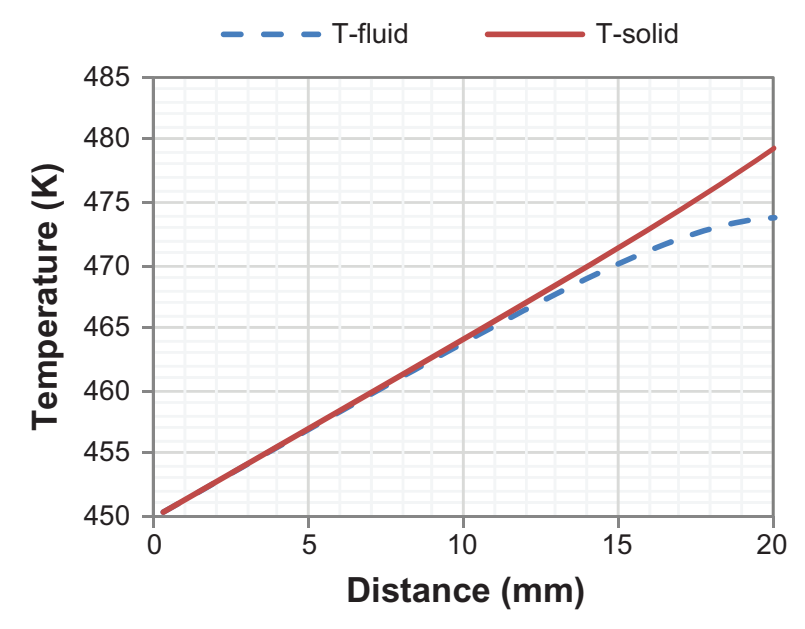

Figure 16 Fluid and solid T profile for $k_{s}=1 \mathrm{~W} / \mathrm{mK}$ with $\mathrm{h}=1 \mathrm{~W} / \mathrm{m}^{2} \mathrm{~K}$. Abbreviations: $\mathrm{T}$, temperature; $\mathrm{h}$, heat transfer coefficient.

observed when the heat flux is completely applied to the fluid boundary, with fluid temperatures going higher than the solid temperatures.

The transfer of heat between fluid and solid domains in the porous model can be viewed as an additional thermal conductance pathway connecting the two parallel resistances of the individual domains (thermal circuit in Figure 11). The resistance of the connection is proportional to the inverse of the heat transfer coefficient used in the porous model. At a higher heat transfer value (low resistance), it is not necessary to split the heat flux between the two domains because the heat will choose the path of least resistance. However, in the cases where interphase heat transfer is low, inaccurate results may be obtained if the heat flux at the boundary is not split as per the procedure mentioned in Equations 20 and 21.

\section{Impact of effective radial fluid conductivity in the porous model}

To verify the assumption of neglecting radial gas phase conductivity, the steady-state heat transfer in the porous model is also solved assuming that the radial conductivity in the gas domain is zero. From Equation 14, it is seen that for zero effective radial conductivity of the gas, $k_{g, e f f}$, the effective solid conductivity, $k_{s, e f f}$, is equal to the effective conductivity of the porous medium, $k_{r, e f f}$ Equations 20 and 21 show that for this case, the total heat flux should be applied to the solid domain boundary. In order to test this assumption under extreme conditions, only two cases (600 cpsi and $300 \mathrm{cpsi})$ with solid thermal conductivity $\left(k_{s}=0.1 \mathrm{~W} / \mathrm{mK}\right)$ are simulated. As seen in Figure 9, the radial gas conductivity is higher than the solid conductivity under these conditions. Surprisingly, the steady-state temperature profile for 
this porous simulation is similar to the porous model profile obtained when the radial thermal conductivity of the gas is not zero. Based on this observation, it is proposed that for steady-state analysis, the solid and fluid domain thermal conductivity can be chosen arbitrarily as long as Equations 14, 20 , and 21 are satisfied.

The above effect is also evident in some of the models published previously. Zygouarkis ${ }^{9}$ and Baba et al, ${ }^{15}$ in their multidimensional models, considered an effective radial conductivity for solids and neglected the gas conductivity. They applied all boundary heat flux on the solid phase energy equation. This is consistent with the proposed Equations 14, 20, and 21. Conversely, in 3D CFD models, ${ }^{1,16}$ the external heat loss boundary condition is applied on mat, which is connected to the solid substrate with an effective radial conductivity. However, finite gas conductivity is also considered with the adiabatic gas phase boundary. This approach is valid as long as the gas conductivity is small compared to the effective conductivity, or if the heat transfer between the two phases is high.

\section{Model validation}

The effective conductivity and the boundary condition derived in the sections above are applied to the continuum representation of the converter model described by Equations 1 to 5 . Both the porous and channel models described above are simulated using the boundary and inlet conditions given in Table 1. The transient response of the monolith to a step change in inlet temperature is obtained for both adiabatic and convective heat loss boundary conditions. Temperature profiles predicted from similar simulations using a one-dimensional (1D) monolith model are also included for comparison. ${ }^{21}$

\section{Results and discussion}

Adiabatic boundary condition

The transient profile of area averaged fluid and solid temperatures is compared at two cross-sectional locations for both the channel and porous models. Figure 17 shows the

Table I Simulation condition

\begin{tabular}{ll}
\hline Mass flow rate & $\mathrm{I} 00 \mathrm{~g} / \mathrm{second}$ \\
Inlet velocity & $5.67 \mathrm{~m} / \mathrm{second}$ \\
Inlet temperature & $500 \mathrm{~K}$ \\
Outlet pressure & $\mathrm{I} \mathrm{atm}$ \\
Heat transfer coefficient & 0 and $100 \mathrm{~W} / \mathrm{m}^{2} \mathrm{~K}$ \\
Ambient temperature & $300 \mathrm{~K}$ \\
Initial temperature & $300 \mathrm{~K}$ \\
\hline
\end{tabular}

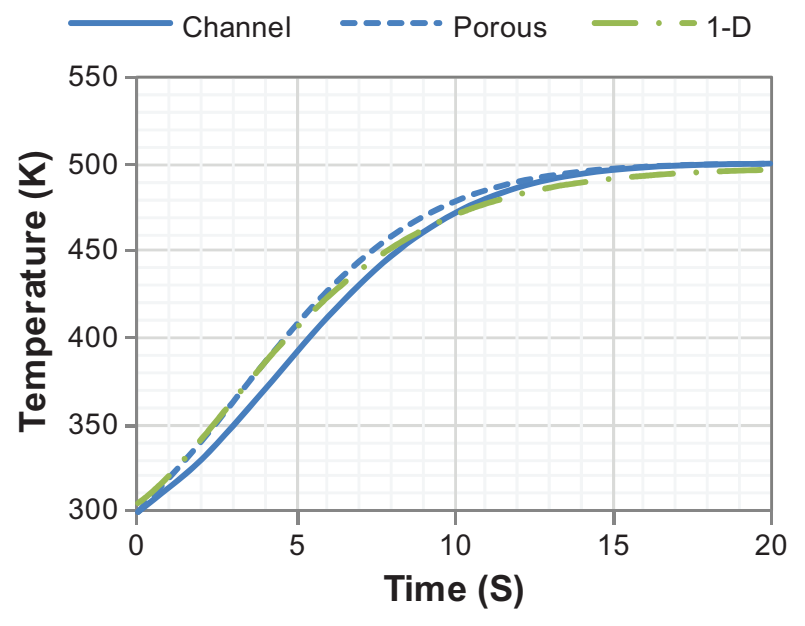

Figure 17 Transient fluid temperature at outlet. Abbreviation: I-D, one-dimensional.

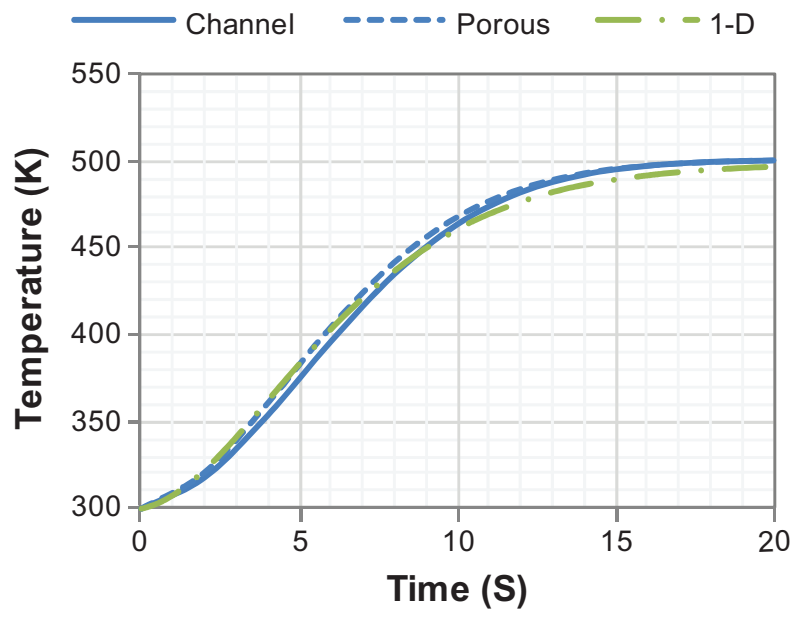

Figure 18 Transient solid temperature at outlet. Abbreviation: I-D, one-dimensional.

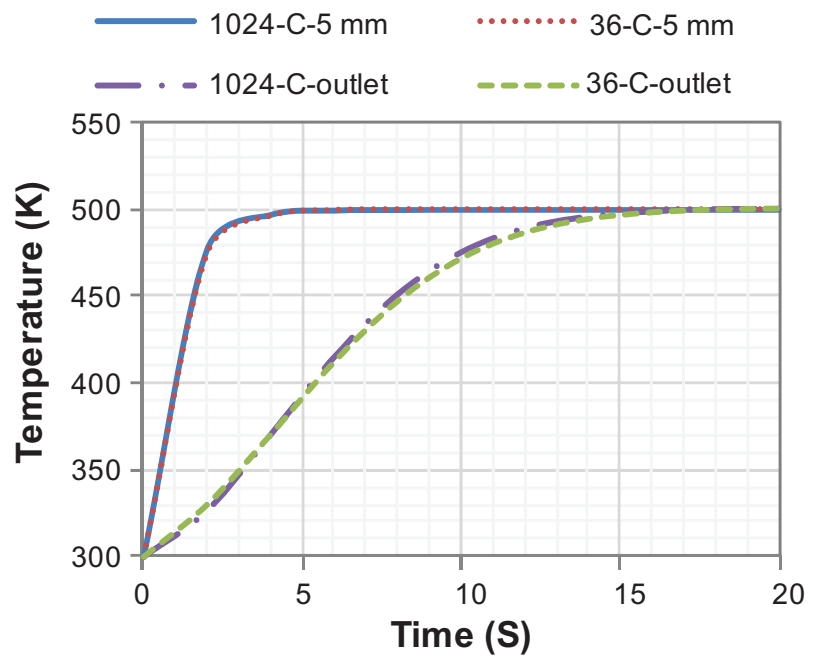

Figure 19 Transient fluid temperature profile. Note: At $5 \mathrm{~mm}$ from inlet and outlet for 36 and 1024 channels. Abbreviation: $C$, channel model. 


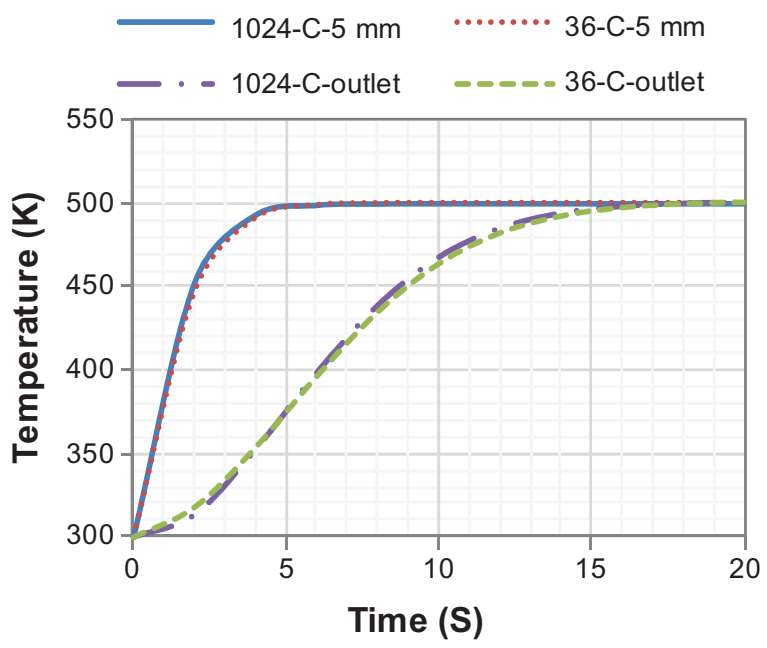

Figure 20 Transient solid temperature profile.

Note: At $5 \mathrm{~mm}$ from inlet and outlet for 36 and 1024 channels.

Abbreviation: $\mathrm{C}$, channel model.

fluid temperature profile at outlet. Figure 18 shows the transient solid temperatures at outlet. The porous model is a good approximation for the channel model. For the adiabatic case, no radial temperature variation is expected, and therefore the results are also in close agreement with those corresponding to the $1 \mathrm{D}$ model results. The transient temperature profiles in the channel model for 36 and 1024 channels are compared in Figures 19 and 20. A good match between the two cases indicates that for the adiabatic boundary condition, 36 channels adequately represent the thermal behavior of full converter.

\section{Heat loss boundary condition}

Transient fluid temperatures for the channel, porous, and 1D models are compared in Figure 21. The solid temperatures

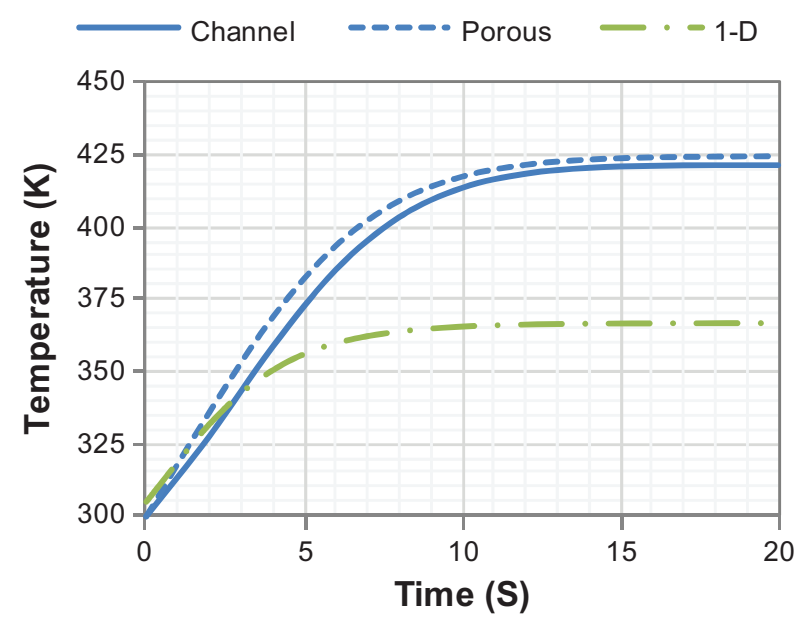

Figure 21 Transient fluid temperature profile at outlet for 36 channels. Abbreviation: I-D, one-dimensional.

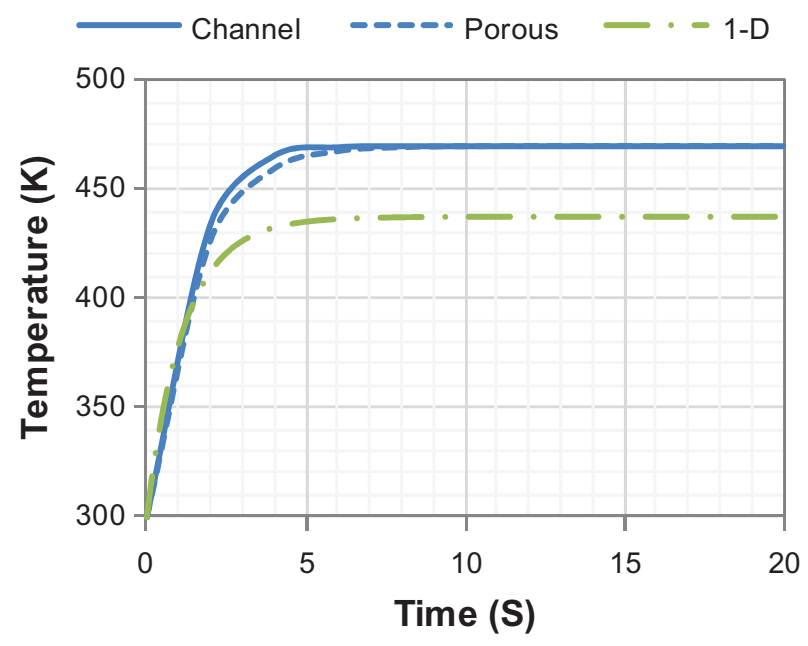

Figure 22 Transient solid temperature profile at a section $5 \mathrm{~mm}$ from inlet for 36 channels.

Abbreviation: I-D, one-dimensional.

are compared in Figures 22 and 23. The 1D model results are obtained by simulating a domain size equivalent to 36 channels. The porous and channel model predictions are close, but the steady-state 1D temperatures are much lower indicating that the 1D model has a higher effective heat loss. In the absence of radial temperature variation, the net heat loss is calculated at an average converter temperature, which will be higher than the converter temperature at the boundary. Figures 24 through 26 show the transient temperature profiles of the fluid and solid regions from the model for 1024 channels. In these graphs, the $1 \mathrm{D}$ model results are obtained using a domain size representing 1024 channels. The porous model results are comparable to the channel model, but the 1D model predicts a lower steady-state temperature.

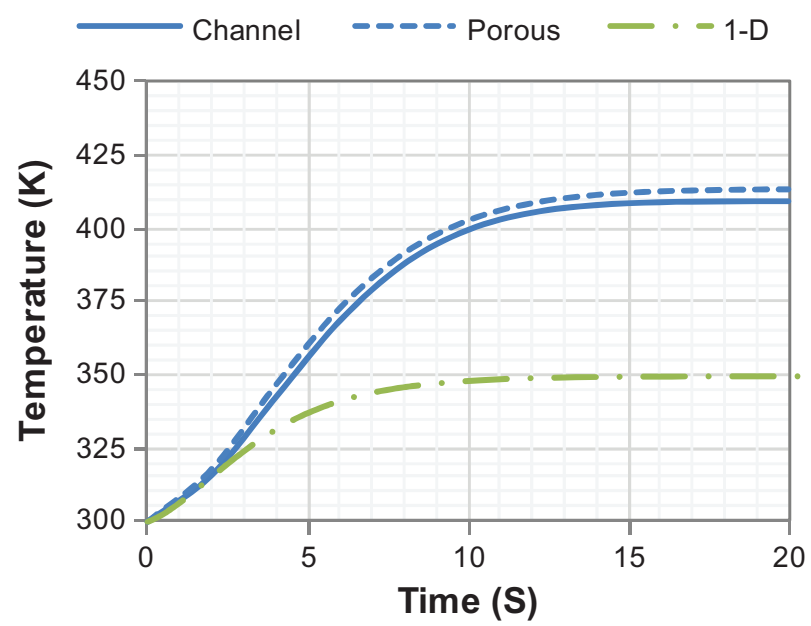

Figure 23 Transient solid temperature profile at outlet for 36 channels. Abbreviation: I-D, one-dimensional. 


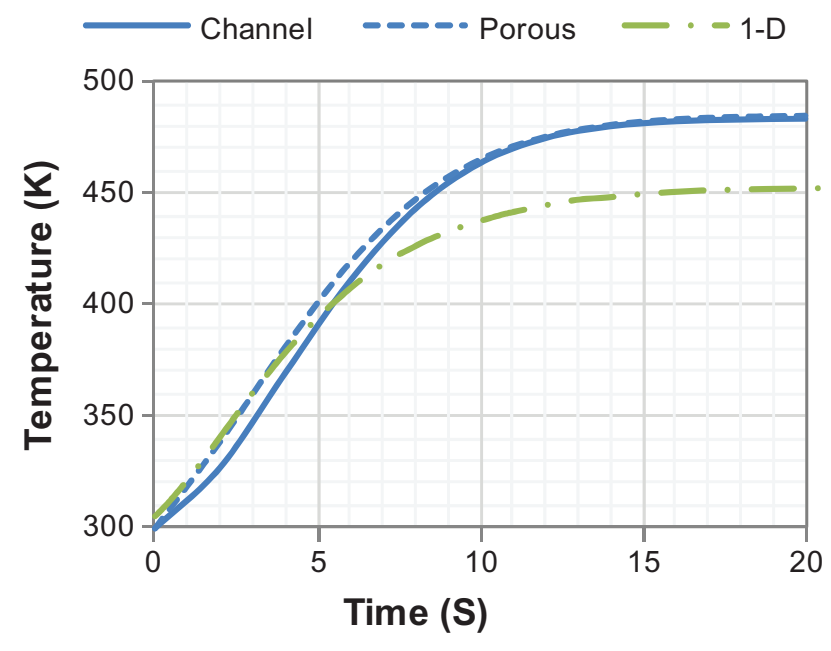

Figure 24 Transient fluid temperature profile at outlet for 1024 channels. Abbreviation: I-D, one-dimensional.

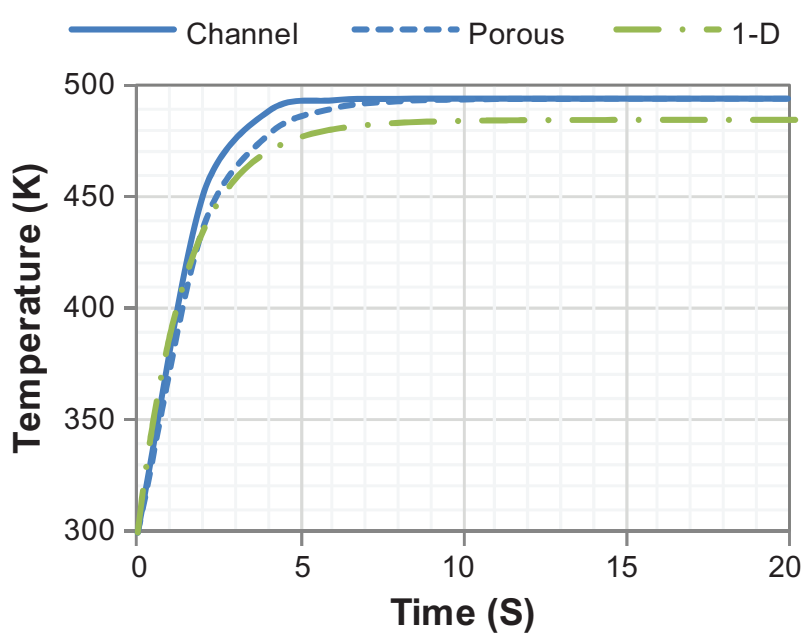

Figure 25 Transient solid temperature profile at a section $5 \mathrm{~mm}$ from inlet for 1024 channels.

Abbreviation: I-D, one-dimensional.

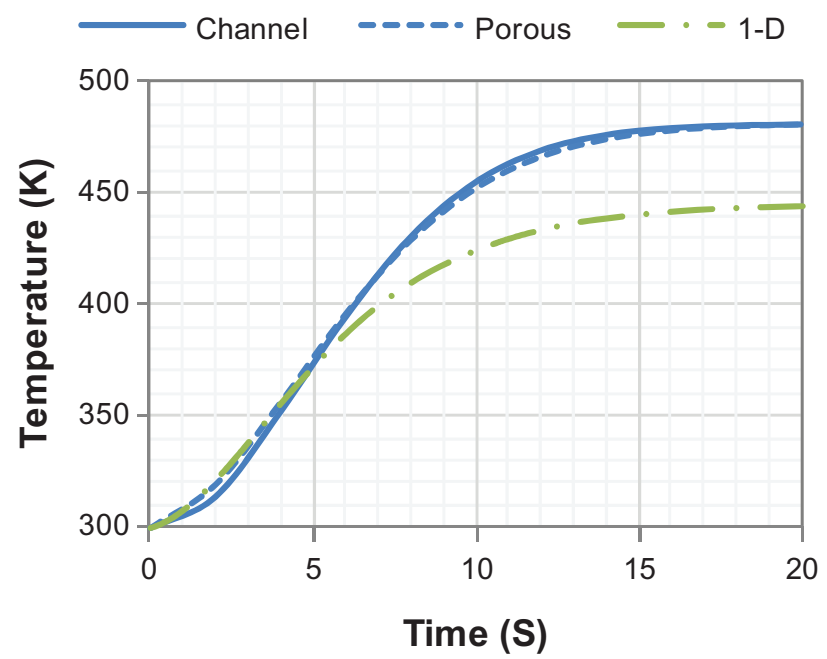

Figure 26 Transient solid temperature profile at outlet for 1024 channels. Abbreviation: I-D, one-dimensional.

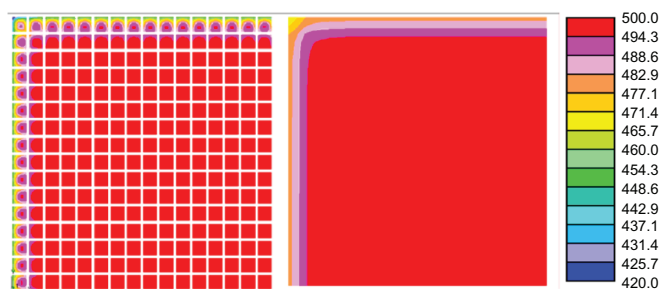

Figure 27 Fluid temperature $(\mathrm{K})$ at 6 seconds.

Note: Contour at a $5 \mathrm{~mm}$ section in the channel model (left) and in the porous model (right).

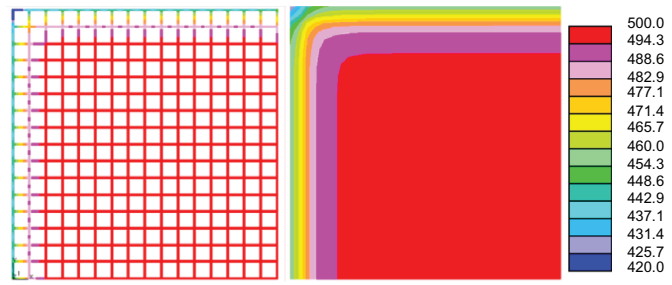

Figure 28 Solid temperature $(K)$ at 6 seconds.

Note: Contour at a $5 \mathrm{~mm}$ section in the channel model (left) and in the porous model (right).

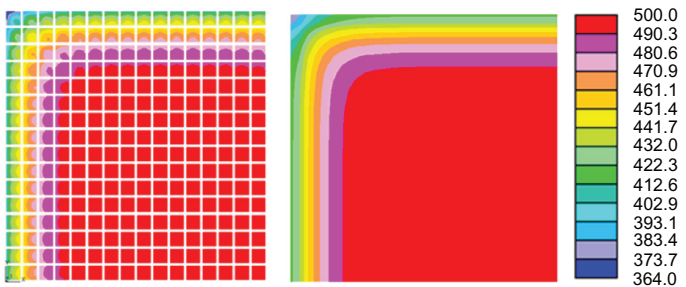

Figure 29 Fluid temperature $(\mathrm{K})$ at 20 seconds.

Note: Contour at outlet in the channel model (left) and in the porous model (right).

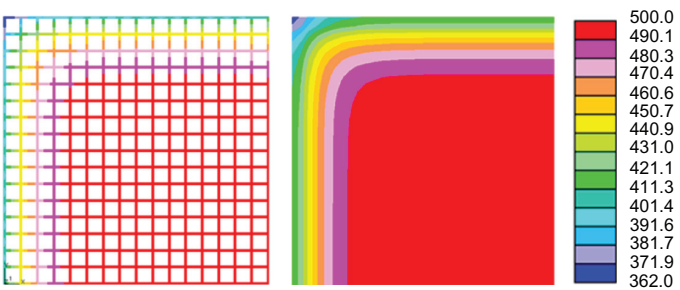

Figure 30 Solid temperature $(\mathrm{K})$ at 20 seconds.

Note: Contour at outlet in the channel model (left) and the porous model (right).

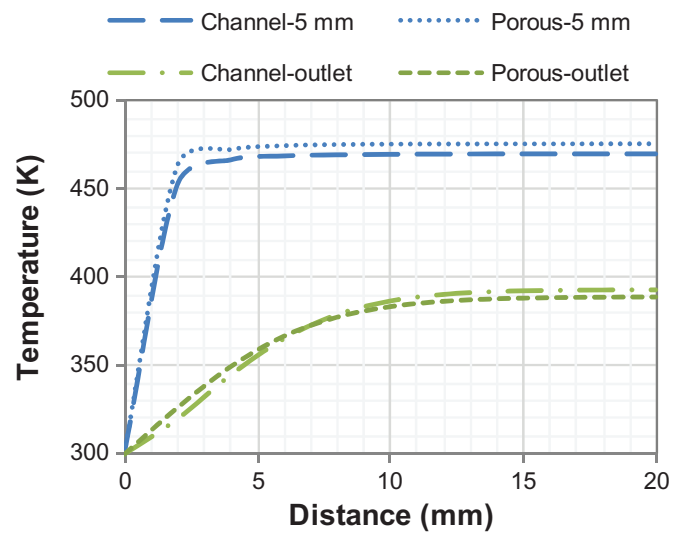

Figure 3 I Fluid temperature profile in a channel near the wall boundary. 


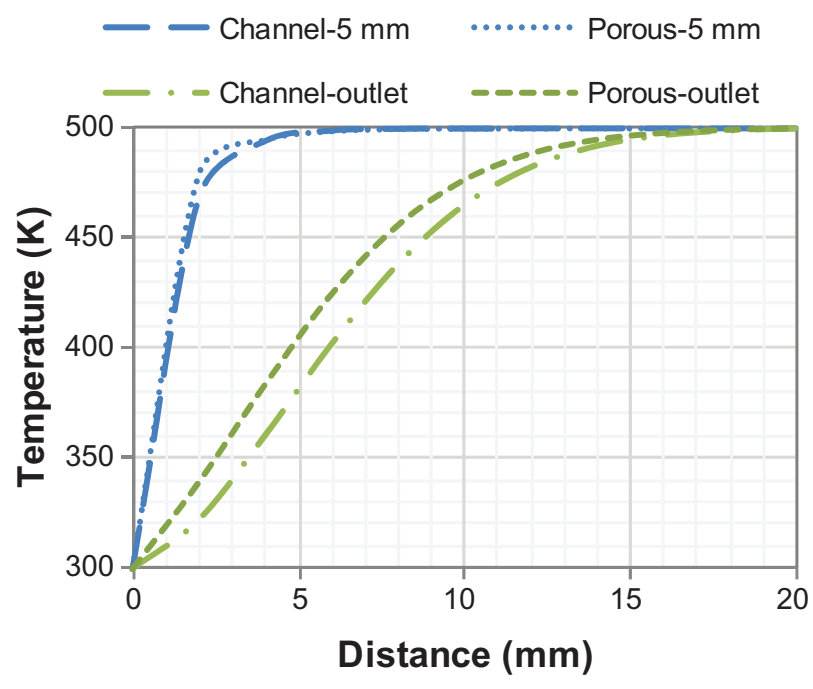

Figure 32 Fluid temperature profile in a channel near the symmetry plane.

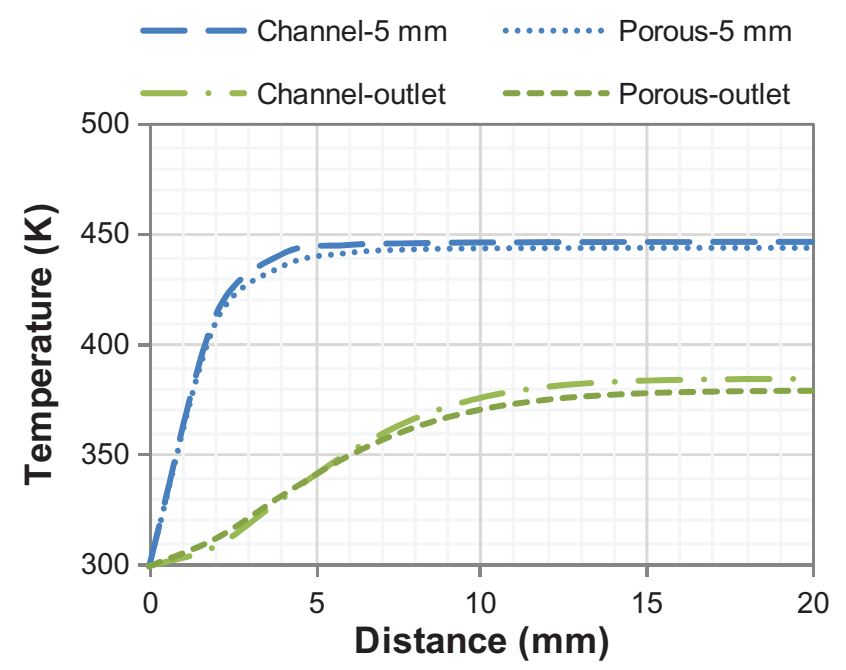

Figure 33 Solid temperature profile in a channel near the wall boundary.

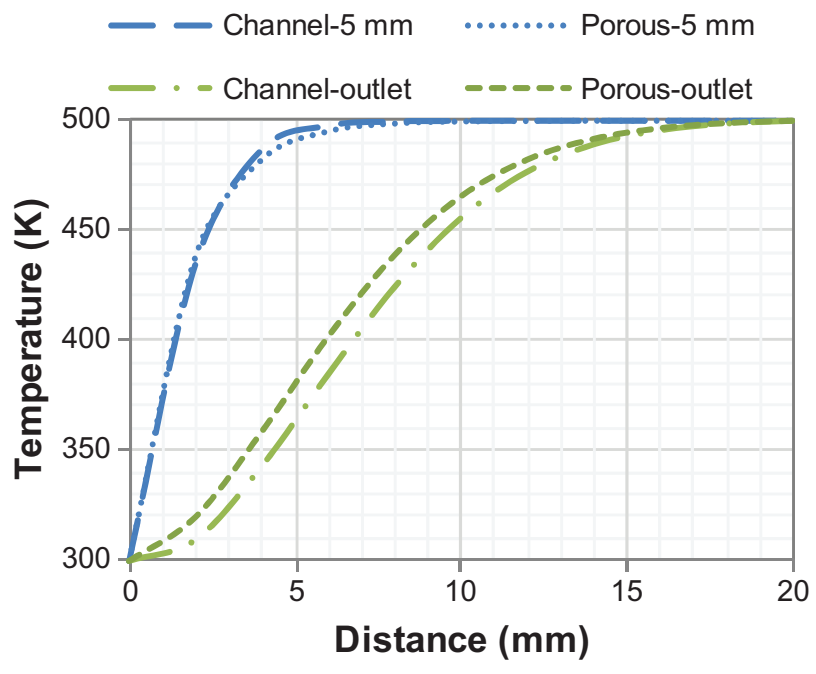

Figure $\mathbf{3 4}$ Solid temperature profile in a channel near the symmetry plane.
The steady-state temperature (at 20 seconds) predicted by models with 1024 channels is higher than that predicted by the models with 36 channels. A higher external surface-to-volume ratio with 36 channels amplifies the effect of heat loss leading to lower temperature predictions. Kumar and Mazumdar ${ }^{3}$ arrived at similar conclusions while modeling methane combustion over converters with 57 and 293 channels. In addition, the $1 \mathrm{D}$ prediction is closer to the CFD prediction when 1024 channels are simulated. Cross-sectional temperature contours in the channel and porous models (Figures 27 to 30 ) show that the effect of heat loss is limited to 2-3 channels near the heat loss boundary. In a smaller model with 36 channels, this has a larger impact on average temperatures, and the radial averaging of heat loss in the 1D model is unable to capture this effect. It is expected that for a typical converter, which will have more than 10000 channels, 1D prediction will be closer to the CFD prediction.

The cross-sectional temperature contours also show that the radial temperature variation in the porous model representation is similar to that observed in the channel model at 6 seconds and 20 seconds after the step change in the inlet temperature. This similarity between the porous and channel models is also highlighted in the transient temperature profile in the channels near the wall and symmetry boundary. The temperature profiles at these two locations in both the channel and porous models are compared in Figures 31 to 34. The transient temperature response predicted by the porous model near the wall and symmetry plane is within $6^{\circ} \mathrm{C}$ of that observed in the channel model. Given these approximations, this is a reasonable validation of the proposed porous model representation.

\section{Conclusions}

The Star-CD porous model is modified to develop a continuum model of the monolith with square channels. The heat transfer in channels is modeled by solving separate energy balance in the porous and solid domain linked through a heat transfer source term. To estimate the effective radial thermal conductivity of each domain, the heat transfer in 1024 square channels is analyzed by direct simulation in Star-CD. The steady-state temperature profile across the 1024 channels shows that effective radial thermal conductivity can be estimated using a thermal resistance network. The resistance network reveals that the solid and fluid regions provide parallel pathways for heat conduction. Further, a methodology is also developed to estimate the effective thermal conductivity of each domain based on the total effective conductivity of the channels. 
It is also shown that an accurate porous model representation of the channels requires the heat flux boundary condition to be split between the fluid and solid domains because the energy balance is solved separately. The split is based on the resistance each domain provides for transverse heat transfer. The consistent treatment of boundary heat flux is important in cases where the interphase heat transfer is low. For cases with a high heat transfer coefficient, the heat flux can be applied in any domain.

For validation, a continuum model of a 1024-channel converter is developed. It is shown that this model can predict the radial variation in temperature to within $6^{\circ} \mathrm{C}$ of that obtained by directly simulating the actual channels. Comparison of results from the 36-channel and 1D models show that the heat transfer problem does not scale with size, and that the full monolith domain should be considered for accurate temperature predictions. The direct simulation of 1024 square channels also shows that the effect of heat loss at the wall is limited to 2-3 channels near the wall.

\section{Disclosure}

The authors report no conflicts of interest in this work.

\section{References}

1. Jeong SJ, Kim WS. A new strategy for improving the warm-up performance of a light-off auto-catalyst for reducing cold-start emissions. Proceedings of the Institution of Mechanical Engineers, Part D: Journal of Automobile Engineering. 2001;215(11):1179-1196.

2. Kolaczkowski ST, Crumpton P, Spence A. Modelling of heat transfer in non-adiabatic monolithic reactors. Chem Eng Sci. 1988;43(2): 227-231.

3. Kumar A, Mazumder S. Toward simulation of full-scale monolithic catalytic converters with complex heterogeneous chemistry. Computers and Chemical Engineering. 2010;34(2):135-145.

4. Clarkson RJ, Benjamin SF, Jasper TS, Girgls NS. An integrated computational model for the optimization of monolith catalytic converters. SAE. 1993;931071.

5. Jeong S, Kim T. CFD investigation of the 3-dimensional unsteady flow in the catalytic converter. Proceedings of the International Congress and Exposition; Detroit, MI; 1997. Detroit, MI: SAE. 971025;1997: $75-87$.
6. Star-CD. STAR-CD Users Guide and Methodology, version 3.26. Computational Dynamics Ltd, New York, NY; 2007.

7. Chen DKS, Bissett EJ, Oh SH, Van Ostrom DL. A three-dimensional model for the analysis of transient thermal and conversion characteristics of monolithic catalytic converters. SAE. 1988;880282.

8. Jeong SJ, Kim WS. A numerical approach to investigate transient thermal and conversion characteristics of automotive catalytic converter. SAE. 1998;980881.

9. Zygourakis K. Transient operation of monolith catalytic converters: a two-dimensional reactor model and the effects of radially nonuniform flow distributions. Chem Eng Sci. 1989;44(9):2075-2086.

10. Liu YH. Equivalent Continuum Models for Nonadiabatic Monolith Catalytic Reactors [dissertation]. Houston, TX: Rice University; 1985.

11. Chakravarthy VK, Conklin JC, Daw CS, D'Azevedo EF. Multi-dimensional simulations of cold-start transients in a catalytic converter under steady inflow conditions. Appl Catal A Gen. 2003; 241(1-2):289-306.

12. Groppi G, Tronconi E. Continuous vs discrete models of nonadiabatic monolith catalysts. Chem Eng Sci. 2000:55.

13. Hayes RE, Rojas A, Mmbaga J. The effective thermal conductivity of monolith honeycomb structures. Catalysis Today. 2009;147 Suppl; S113-S119.

14. Sahraoui M, Kaviany M. Direct simulation vs volume-averaged treatment of adiabatic, premixed flame in a porous medium. Int J Heat Mass Transf. 1994;37(18):2817-2834.

15. Baba N, Ohsawa K, Sugiura S. Numerical approach for improving the conversion characteristics of exhaust catalysts under warming-up condition. SAE Transactions. 1996;105(4):2064-2079.

16. Chung $C$, Geer L, Rajadurai $S$. Numerical simulation and experimental validation of the catalytic converter cool down process. SAE. 2000; 2000-01-0204.

17. Kays WM, London AL. Compact Heat Exchangers. Malabar, FL, USA: Krieger Publishing Company; 1984.

18. Jeong SJ, Kim WS. A study on the optimal monolith combination for improving flow uniformity and warm-up performance of an autocatalyst. Chem Eng Process. 2003;42(11):879-895.

19. Barin O, Knacke O, Kubaschewski O. Thermochemical Properties of Inorganic. Substances: Supplement. New York: Springer-Verlag; 1977.

20. Todd B, Young JB. Thermodynamic and transport properties of gases for use in solid oxide fuel cell modelling. J Power Sources. 2002;110(1): 186-200.

21. Rao SK, Imam R, Ramanathan K, Pushpavanam S. Sensitivity analysis and kinetic parameter estimation in a three way catalytic converter. Ind Eng Chem Res. 2009;48(8):3779-3790.

\section{Publish your work in this journal}

Energy and Emission Control Technologies is an international, peer-reviewed, open access journal publishing original research, reviews, editorials and commentaries on developing technologies to optimize energy production and control of emissions. The manuscript management system is completely online and includes

\section{Dovepress}

a very quick and fair peer-review system, which is all easy to use. Visit http://www.dovepress.com/testimonials.php to read real quotes from published authors. 